\title{
Estrategia en la determinación del factor de forma de radiación ilustrado con el sistema plano-esfera
}

\author{
Héctor Armando Durán Peralta* \\ Orlando Hernández Fandiño** \\ Artículo recibido: 18-10-2005 y aprobado:28-09-2006
}

\begin{abstract}
Strategy in the determination of the radiation form factor illustrated by the plane-sphere system
\end{abstract}

Resumen: En el diseño de hornos y dispositivos que calientan o enfrían sistemas mediante radiación se necesita calcular el factor de forma, el cual permite determinar la transferencia de calor por radiación entre superficies que estén a distinta temperatura. Generalmente, en los textos que tratan de transferencia de calor se deduce y muestra la ecuación para obtener el factor de forma entre dos superficies que intercambian calor por radiación para configuraciones muy sencillas, pero no se enseña cómo se hace el cálculo para geometrías y configuraciones un poco más complejas. Mediante este documento se pretende dar-con fines netamente académicos- una estrategia para calcular el factor de forma de otras configuraciones, mostrando dicha estrategia al desarrollar un caso particular, como el cálculo del factor de forma plano-esfera.

Palabras clave: factor de forma, transferencia de calor, radiación, sistema plano-esfera.
Abstract: The form factor must be calculated when designing ovens and mechanisms heating or cooling systems by radiation leading to determining heat transfer by radiation between surfaces which are at different temperatures. Heat transfer texts generally deduce and show the equation for obtaining the form factor between two surfaces which exchange heat by radiation for very simple configurations, but do not show slightly more complex geometries and configurations are calculated. This document seeks highly with ends academics to give a strategy to continue of how to calculate the factor in way of other configurations, showing this strategy to continue when developing a particular case, the calculation of the factor in way plane-sphere.

Key words: Form factor, heat transfer, radiation, planesphere system.

\footnotetext{
Departamento de Ingeniería Química, Facultad de Ingeniería, Universidad Nacional de Colombia, Bogotá. haduranp@unal.edu.co.

** Departamento de Química, Facultad de Ciencias, Universidad Nacional de Colombia, Bogotá.

ohernandezf@unal.edu.co.
} 


\section{Introducción}

La transferencia de calor es un fenómeno de gran aplicación en la industria y que ha recibido bastante atención desde el punto de vista teórico-práctico. En el diseño de hornos y dispositivos que calientan o enfrían sistemas mediante radiación se necesita calcular el factor de forma, el cual permite determinar la transferencia de calor por radiación entre superficies que estén a distinta temperatura. Generalmente, en los textos de transferencia de calor se deduce y muestra la ecuación para obtener el factor de forma entre dos superficies que intercambian calor por radiación para configuraciones muy sencillas, pero no se enseña cómo se hace el cálculo para geometrías y configuraciones un poco más complejas. Muchos de los estudiantes que han cursado la asignatura o asignaturas de transferencia de calor quedan con la impresión de que los factores de forma se calculan sin ningún problema. Otros estudiantes preguntan por qué en los textos no se muestra cómo se calcula el factor de forma para configuraciones un poco más reales o más complejas. Por eso en este documento, mediante la solución de un caso aparentemente sencillo (cálculo del factor de forma de la configuración plano-esfera), se pretende responder en parte a esta inquietud, a la vez que se proponen lineamientos generales sobre una estrategia de cálculo con fines netamente académicos.

Entre los mecanismos de transferencia están la conducción, convección y radiación, mecanismo este último que ha merecido, relativamente, menor atención en la literatura, a pesar de sus importantes aplicaciones industriales en hornos de arco, colectores y hornos solares, enfriamiento de cuerpos incandescentes (tuberías, piezas de fundición, etc.) y cálculo de barreras de protección.

La radiación es un fenómeno que sucede en todos los cuerpos sólidos y corresponde a la emisión espontánea de ondas electromagnéticas, en todas las longitudes de onda, pero que presenta un intervalo de emisión (de longitudes de onda) preferencial, que depende de la temperatura del cuerpo. Por esta razón, un cuerpo a temperatura ambiente emite principalmente radiaciones en el espectro no visible, mientras que a altas temperaturas $\left(500 \mathrm{a} 2000^{\circ} \mathrm{C}\right.$ ) predomina el espectro visible.

Cuando se tienen dos superficies, cada una emite energía radiante (según su nivel de temperatura) hacia los alrededores, y parte de ella la intercepta la otra. La relación entre la energía interceptada por una superficie y la total emitida por la otra es lo que se conoce como factor de visión o factor de forma. Por tal motivo, los factores de forma dependen de la geometría y configuración entre las superficies (1), (2), y de los ángulos, respecto de la normal de la superficie, con que se emitan las radiaciones en cada punto. Estos ángulos del presente documento se denominarán ángulos de visión a lo largo.

El cálculo del flujo de calor por radiación se hace, en estos casos, mediante ecuaciones de balance de energía que incluyen el factor de forma, el cual puede calcularse a partir de la ecuación 1 (2).

$F_{1-2}=\left(\frac{1}{\pi A_{1}}\right) \int_{A_{1}} \int_{A_{2}} \frac{\cos \theta_{1} \cos \theta_{2}}{r^{2}} d A_{1} d A_{2}$ 
Con este artículo se pretende, en primer lugar, mostrar una estrategia para calcular el factor de forma ilustrado con un caso particular (plano-esfera), y en segundo término, solucionar la ecuación del factor de forma, para el caso del calentamiento de la pared de un horno mediante un arco esférico, aplicando el método de Montecarlo.

\section{Desarrollo del modelo}

Como modelo de análisis para calcular el factor de forma se ha escogido el sistema compuesto por una superficie esférica y un plano, que se esquematiza en la figura 1.

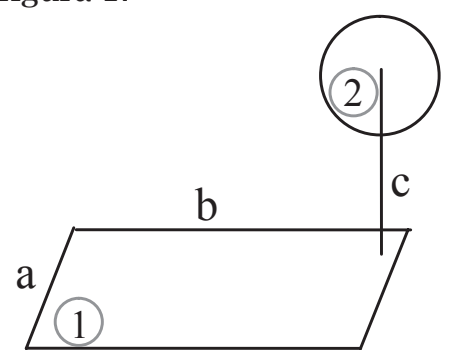

Figura 1. Sistema plano-esfera.

En este esquema, la superficie plana 1 de dimensiones $a \times b$, representa la pared del horno; la superficie esférica 2, de radio $R$, representa el arco voltaico, y la separación entre ellas se designa por $c$. Para solucionar la ecuación 1 se requiere, en cada caso particular, determinar los límites y la relación entre las variables que intervienen. La estrategia para calcular el factor de forma se puede resumir en tres pasos:

1. Determinar los límites de las integrales.

2. Determinar los cosenos de los ángulos de visión en términos de las variables de integración.

3. Solucionar de la integral múltiple mediante un método matemático adecuado, que en nuestro caso será el método de Montecarlo.

Para la solución nos basaremos en el esquema presentado en la figura 2 , donde un punto sobre la esfera se denota como $p(x, y, z)$ y uno sobre el plano como $p_{i}\left(x_{i}, y_{i}, 0\right) ; r$ es la distancia recorrida por el rayo emitido desde el punto emisor $p_{i}\left(x_{i}, y_{i}, 0\right)$ hasta el receptor $p(x, y, z)$ o viceversa; $\theta_{1}$ es el ángulo de visión formado entre la normal a la superficie del plano en el punto $p(x, y, z)$ y $r ; \theta_{2}$ es el ángulo de visión formado entre la normal a la superficie esférica en el punto $(x, y, z)$ y la línea $r$.

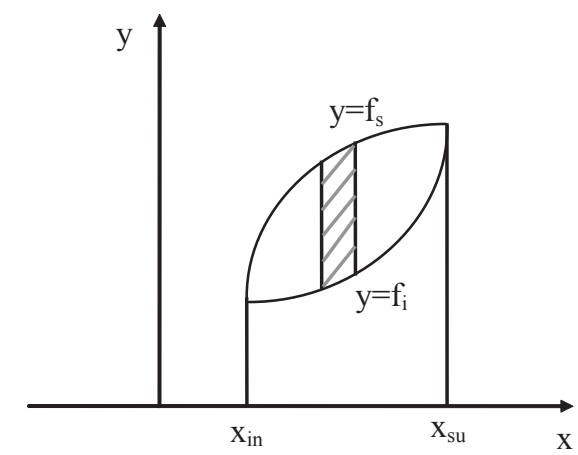

Figura 2. Puntos frontera de visión: a): Los puntos situados más allá de la base del cono (circunferencia), sobre la esfera, no ven el punto Pi(xi , yi, 0) sobre el plano. b): Proyección de la base del cono sobre el plano, el cual da una elipse. 
Las coordenadas $(x, y, z)$ corresponden al dominio sobre la esfera $G$ de radio $R$ y centro $(h, k, l)$, cuya ecuación es:

$G(x, y, z)=(x-h)^{2}+(y-k)^{2}+\left(z-l^{2}\right)-R^{2}=0$

Un elemento de área en el plano, calculado en términos de $x_{i}, y_{i}$, viene dado por:

$$
d A_{1}=d x_{i} d y_{i}
$$

y un elemento de área en la superficie esférica calculado en términos de $x, y$ viene dado por:

$$
d A_{2}=\frac{R d x d y}{\sqrt{R^{2}-(x-h)^{2}-(y-k)^{2}}}
$$

Sustituyendo los elementos de área $d A_{1}, d A_{2}$ en la integral, resulta en definitiva una integral cuádruple evaluada sobre una región $\mathbf{R}$ :

$F_{1-2}=\left(\frac{1}{\pi A_{1}}\right) \iiint \int_{R} \frac{R \cos \theta_{1} \cos \theta_{2} d x d y d x_{i} d y_{i}}{r^{2} \sqrt{R^{2}-(x-h)^{2}-(y-k)^{2}}}$

\section{Determinación de los límites de las integrales}

Si se toma un punto $p_{1}\left(x_{i}, y_{i}, 0\right)$ del plano, éste emite energía radiante en todas las direcciones con la misma intensidad, o sea, en forma difusa ideal. La primera integral de derecha a izquierda de la ecuación 1 representa la parte de esta energía que emite el punto $\mathrm{p}_{\mathrm{i}}$ y que intercepta la esfera. En la figura 2, el ángulo sólido (w) abarca la energía emitida desde el punto $\mathrm{p}_{\mathrm{i}} \mathrm{y}$ forma un cono generado por los rayos emitidos desde $\mathrm{p}_{\mathrm{i}}$, que llegan a la esfera, quedando el cono tangente a la esfera, es decir, el ángulo sólido abarca el espacio limitado por el cono tangente a la esfera, cuyo vértice es el punto $p_{1}\left(x_{i}\right.$, $y_{i}$, O) situado en el plano. Los límites geométricos para la primera integral en la ecuación 1 estarán dados de acuerdo con la ecuación para $d A_{2}$ siendo ésta la elipse resultante de la proyección de la base del cono tangente (circunferencia) sobre el plano. La ecuación rectangular de la base del cono en el espacio $\mathrm{R}^{3}$ es la resultante de la intersección de la esfera (ecuación 2) y el cono tangente (ecuación 4):

$$
\begin{aligned}
& (x-h)^{2}+(y-k)^{2}+\left(z-l^{2}\right)=R^{2} \\
& \left|\begin{array}{cc}
x & y \\
\left(k-y_{i}\right) & \left(x_{i}-h\right)
\end{array}\right|-l Z=\left|\begin{array}{cc}
x_{i} & y_{i} \\
-k & h
\end{array}\right|+R^{2}-\left(h^{2}+k^{2}+l^{2}\right)
\end{aligned}
$$

De las dos ecuaciones anteriores es posible eliminar $z$ para obtener la ecuación de la proyección de la circunferencia sobre el plano, resultando: 


$$
A x^{2}+B x y+C y^{2}+D x+E y+F=0
$$

Para resolver la ecuación 3 se integra inicialmente con respecto de $y$, que corresponde a la ordenada de los puntos sobre la superficie de la esfera, y en consecuencia los límites quedan en función de $\left(x_{i}, y_{i}, x\right)$ Con base en la ecuación 6 se establecen los límites de integración superior e inferior para $y$, que corresponden a las funciones $f_{i}(x$, $\left.x_{i}, y_{i}\right)$ y $f_{s}\left(x, x_{i}, y_{i}\right)$ definidas como:

$$
\begin{aligned}
& f_{i}\left(x, x_{i}, y_{i}\right)=\alpha-\left(\beta x^{2}+\gamma x+\eta\right)^{1 / 2}: \text { límite inferior para "y" } \\
& f_{s}\left(x, x_{i}, y_{i}\right)=\bar{\alpha}-\left(\bar{\beta} x^{2}+\bar{\gamma} x+\bar{\eta}\right)^{1 / 2} \text { : límite superior para "y" }
\end{aligned}
$$

Donde $\alpha, \beta, \gamma, \eta$ y $\bar{\alpha}, \bar{\beta}, \bar{\gamma}, \bar{\eta}$ dependen de $x_{i}, y_{i}$

Para resolver la segunda integral se deben establecer los límites de $x$, que corresponden a los valores máximo y mínimo de $x$ en la proyección de la base del cono, con vértices en $P_{i}\left(x, x_{i}\right.$, $0)$ sobre la del plano. El tamaño del intervalo de integración para $x$ depende de la proyección de la circunferencia sobre el plano. En la figura $2 \mathrm{~b}$ puede verse que los límites para $x$ son $x_{i n}$, $x_{\text {su }}$, los cuales dependen de la posición del punto $P_{i}\left(x_{i}, y_{i}, 0\right)$. Los demás límites de las integrales no presentan problema. Reemplazando en la ecuación 3, resulta:

$$
\begin{gathered}
\qquad 1-2=\left(\frac{1}{\pi A_{1}}\right) \int_{0}^{b} \int_{0}^{a} \int_{x_{i n}}^{x_{s u}} \int_{f_{i}}^{f_{s}} \frac{R \cos \theta_{1} \cos \theta_{2} d x d y d x_{i} d y_{i}}{r^{2} \sqrt{R^{2}-(x-h)^{2}-(y-k)^{2}}} \\
\text { Donde } \quad f_{i}=f_{i}\left(x, x_{i}, y_{i}\right) ; f_{s}=f_{s}\left(x, x_{i}, y_{i}\right) \\
x_{i n}=x_{i n}\left(x_{i}, y_{i}\right) ; x_{s u}=x_{s u}\left(x_{i}, y_{i}\right)
\end{gathered}
$$

Establecidos los límites de la integral múltiple, el paso siguiente es determinar el coseno de los ángulos de visión.
Determinación de los cosenos de los ángulos de visión $(\cos \theta 1$ y $\cos \theta 2)$ Con base en la figura 3, se establecen geométricamente las siguientes expresiones para los cosenos de los ángulos de visión en función de $x, y, x_{i}, y_{i}$ : 


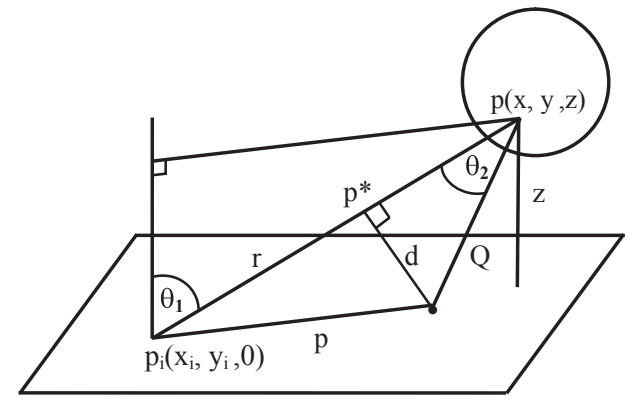

Figura 3. Construcción geométrica para calcular el coseno de los ángulos de visión.

$$
\cos \theta_{1}=\frac{z}{r}
$$

Donde $r=\sqrt{\left(x-x_{i}\right)^{2}+\left(y-y_{i}\right)^{2}+(z-0)^{2}}$

$$
\operatorname{sens} \theta_{2}=\frac{d}{p \varepsilon}
$$

Donde $p \varepsilon$ es la distancia entre los puntos $p$ y $\varepsilon$, que corresponde a la interceptación normal a la esfera en el punto $p$ con el plano. Por tanto,

$$
\cos \theta_{2}=\sqrt{1-\left(\frac{d}{p \varepsilon}\right)^{2}}
$$

Y la distancia entre los dos puntos será:

$$
p \varepsilon=\sqrt{\left(x-x_{\varepsilon}\right)^{2}+\left(y-y_{i \varepsilon}\right)^{2}+(z-0)^{2}}
$$

Si se designa como $d$ la distancia entre $p^{*}$, que es el pie de la perpendicular a $r$ que pasa por $\varepsilon$ y aplicamos la fórmula de distancia entre dos puntos (4), se tendrá:

$d=\sqrt{\left(x^{*}-x_{\varepsilon}\right)^{2}+\left(y^{*}-y_{\varepsilon}\right)^{2}+\left(z^{*}-z_{\varepsilon}\right)^{2}}$

Según se muestra en la figura- $3, \mathrm{z}_{\varepsilon}$ está en el plano horizontal coordenado, el cual tiene posición $Z=0$, por lo que $Z_{\varepsilon}=0$.

Es importante observar que los ángulos $\theta_{1}$ y $\theta_{2}$ que aparecen en la figura 3 no están en un mismo plano.

El cálculo de las coordenadas del punto $\varepsilon$ y $p^{*}$ puede realizarse utilizando conceptos de geometría analítica en el espacio, los cuales están consignados en el apéndice A. Estas coordenadas son:

$$
\begin{aligned}
& x_{\varepsilon}=x-\frac{(x-h) z}{(z-l)} \\
& y_{\varepsilon}=y-\frac{(y-k) z}{(z-l)}
\end{aligned}
$$

$Y Z_{\varepsilon}$ que ya se dijo cómo se determinó, dando el valor

$$
z_{\varepsilon}=0
$$

Al calcular las coordenadas del punto $p^{*}\left(x^{*}, y^{*}, z^{*}\right)$,éstas quedan expresadas implícitamente con el siguiente sistema de ecuaciones:

$$
\begin{gathered}
z x^{*}-\left(x-x_{i}\right) z^{*}=-\left(z x_{i}\right) \\
z y^{*}-\left(y-y_{i}\right) z^{*}=-\left(z y_{i}\right) \\
-z\left(x-x_{i}\right) x^{*}-z\left(y-y_{i}\right) y^{*}-z^{2} z^{*}=z\left(x-x_{i}\right) x_{\varepsilon}-z\left(y-y_{i}\right) y_{\varepsilon}
\end{gathered}
$$


La solución analítica de estas tres ecuaciones dará expresiones voluminosas para $x^{*}, y^{*}, z^{*}$, por lo cual se solucionan numéricamente dentro del desarrollo del programa.

Determinados los puntos $\varepsilon\left(x_{\varepsilon}\right.$, $\left.y_{\varepsilon}, 0\right), p^{*}\left(x^{*}, y^{*}, z^{*}\right)$ y las distancias $p \varepsilon$ y $d$, que corresponde al segmento $p \varepsilon^{*}$, se pueden calcular los cosenos de los ángulos de visión mediante las expresiones 8 y 9 . Estos cosenos se determinan numéricamente en el programa del cálculo del factor de visión (FVIS).

Una vez expresados los cosenos de los ángulos $\theta_{1}$ y $\theta_{2}$ en función de las variables $x, y, x_{i}, y_{i}$, se remplazan en la ecuación 7 , la cual queda lista para ser integrada y cuya solución dará el factor de forma plano-esfera. Si se efectúan las sustituciones indicadas en la ecuación 7, la expresión bajo la integral múltiple resultaría muy voluminosa, por lo que la integral 7 se resuelve con el programa numéricamente y usando el método de Montecarlo.

\section{Cálculo del factor de visión mediante la integral $F_{1-2}$,empleando el método de Montecarlo (algoritmo del programa FVIS)}

Para evaluar integrales múltiples en dimensiones hasta dos e incluso tres se utilizan métodos como el de Simpson, cuadratura gaussiana, integración de Romberg, etc. (5). La experiencia demuestra que los métodos tradicionales para evaluar integrales múltiples en dimensiones mayores de tres se vuelven muy lentos debido a los numerosos cálculos que hay que efectuar (6). En estos casos se recurre a los métodos probabilísticas, como por ejemplo el método de Montecarlo.
La integral del factor de visión es una integral múltiple en dimensión 4, por lo que su evaluación se efectuará mediante el método de Montecarlo. Para este caso se utilizará la versión de Montecalo del disparo aleatorio hacia un blanco fijo (7).

En la figura 4 aparece el algoritmo de cálculo para determinar el factor de visión del arreglo plano-esfera. En términos generales, el algoritmo permite calcular los límites variables de la integral múltiple, como son: $f_{i}(x$, $\left.x_{i}, y_{i}\right), f_{s}\left(x, x_{i}, y_{i}\right), x_{i n}\left(x_{i}, y_{i}\right), x_{s u}\left(x_{i}\right.$, $\left.y_{i}\right)$; calcula también los puntos $p^{*}$ y $\varepsilon$, que permiten evaluar el coseno de los ángulos de visión. Y finalmente evalúa la integral $F_{1-2}$, que determina el factor de visión.

Este algoritmo corresponde al programa FVIS, mediante el cual se puede calcular el factor de visión de la configuración plano-esfera. Las instrucciones del programa están escritas en Matlab versión 4.0 para Windows*. El programa FVIS, se ejecutó con el fin de calcular $F_{1-2}$ para los parámetros geométricos mostrados en la figura 1 (resultados para diferentes valores de estos parámetros se encuentran en la tabla 1). En el método de Montecarlo se realizan sorteos para calcular la integral, cuyo número se puede fijar en el programa; para los cálculos del factor de visión de acuerdo con los parámetros seleccionados, se fijaron 200 sorteos. Según el factor de visión se calculó el número de sorteos (ss) que quedaron dentro de la región del espacio $\mathrm{R}^{4}$, limitada por la función $F$ (el integrando de la integral $F_{1-2}$ ). La expresión para calcular $F_{1-2}$ fue la siguiente: 


$$
F_{1-2}=(X S U P-X I N) *(Y S U P-Y I N) * U M * \frac{s S}{m \pi A_{1}}
$$

En la figura 5 se graficó el factor de visión $\mathrm{F}_{1-2} \mathrm{VS}$ b/c con los demás parámetros constantes.

\section{Análisis de resultados}

- El método desarrollado permite calcular el factor de visión de la configuración "superficie plana hacia

una superficie esférica", siempre y cuando la proyección ortogonal de la esfera quede contenida en el plano.

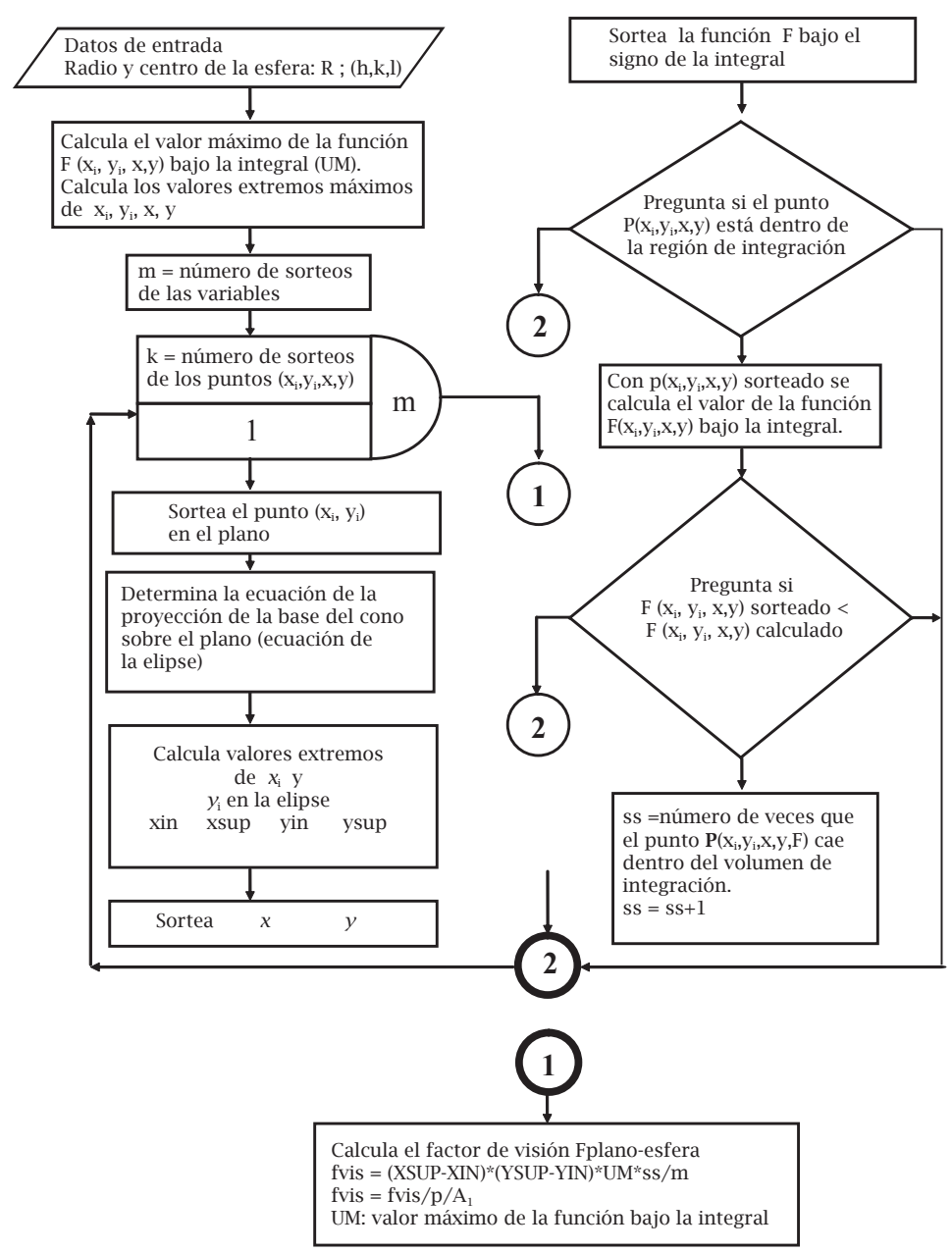

Figura 4. Algoritmo de cálculo para calcular el factor de visión (plano-esfera) mediante el método de Montecarlo 
Tabla 1

\begin{tabular}{|c|c|c|c|c|c|c|c|c|}
\hline $\mathrm{a}$ & $\mathrm{b}$ & $\mathrm{c}$ & $\mathrm{R}$ & $\mathrm{a} / \mathrm{c}$ & $\mathrm{b} / \mathrm{c}$ & $\mathrm{d} / \mathrm{c}$ & $\mathrm{F} 1-2$ & N.o de sorteos \\
\hline 1 & 0,25 & 0,75 & 0,125 & 1,333 & 0,333 & 0,333 & 0,0974 & 200 \\
\hline 1 & 0,75 & 0,75 & 0,125 & 1,333 & 1,000 & 0,333 & 0,1004 & 200 \\
\hline 1 & 1,00 & 0,75 & 0,125 & 1,333 & 1,333 & 0,333 & 0,0856 & 200 \\
\hline 1 & 2,00 & 0,75 & 0,125 & 1,333 & 2,667 & 0,333 & 0,0290 & 200 \\
\hline 1 & 2,50 & 0,75 & 0,125 & 1,333 & 3,333 & 0,333 & 0,0256 & 200 \\
\hline 1 & 3,00 & 0,75 & 0,125 & 1,333 & 4,000 & 0,333 & 0,0259 & 200 \\
\hline
\end{tabular}

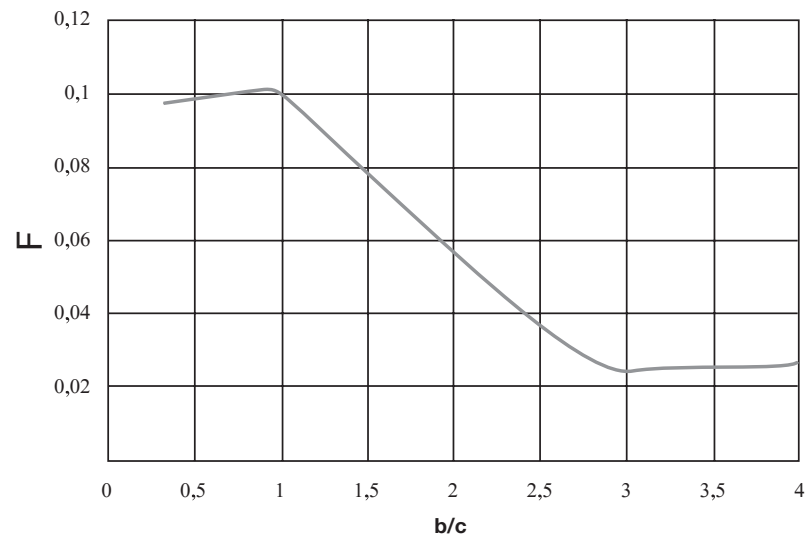

Figura 5. Factor de visión plano-esfera F vs b/c

- En los valores obtenidos se puede apreciar que la exactitud depende del número de sorteos realizados, ya que cuanto menor sea el número de éstos el intervalo de confianza se hace mayor, por lo que diferencias entre tamaños distintos no van a ser tan evidentes. Esto se aprecia en la tabla 1, en la que el primer valor para las dos primeras corridas da menor que el segundo, cosa que no debe ocurrir, pero en realidad los dos se encuentran dentro del mismo intervalo de confianza.

- La convergencia del método con el número de sorteos empleado es lenta, pero se espera que mejore al incrementar el número de sorteos, como lo plantea el método. $\boldsymbol{\Delta}$

\section{Notación}

$F_{1-2}$ : Factor de forma de radiación desde la superficie-1 hacia la superficie-2.

$A_{1}$ : Área de superficie de emisión difusa o receptora de radiación $\left(\mathrm{m}^{2}\right)$.

$A_{2}$ : Área de superficie receptora de radiación $\left(\mathrm{m}^{2}\right)$. 
$\theta_{1}$ : Ángulo de visión formado entre la normal a la superficie del plano y el vector $\vec{r}$.

$\theta_{2}$ : Ángulo de visión formado entre la normal a la superficie de la esfera y el vector $\vec{r}$.

$\vec{r}$ : Vector formado al unir el punto $\left(\mathrm{x}_{\mathrm{i}}, \mathrm{y}_{\mathrm{i}}, 0\right)$ del plano y el punto $(\mathrm{x}, \mathrm{y}, \mathrm{z})$ en la esfera.

$x$ : Abscisa coordenada sobre un punto genérico de la esfera (m).

$y$ : Ordenada sobre un punto genérico de la esfera (m).

$z$ : Traza coordenada sobre un punto genérico de la esfera $(\mathrm{m})$.

$x_{i}$ : Abscisa coordenada sobre un punto genérico del plano (m).

$y_{i}$ : Ordenada sobre un punto genérico del plano (m).

$R$ : Radio de la esfera (m).

$h$ : Abscisa correspondiente al centro de la esfera (m).

$k$ : Coordenada correspondiente al centro de la esfera (m).

l: Traza coordenada correspondiente al centro de la esfera (m).

$f$ : Límite inferior para $y$ sobre la superficie esférica (m).

$f_{s}$ : Límite superior para $y$ sobre la superficie esférica.

$x_{i n}$ : Límite inferior para $x$ sobre la esfera $(\mathrm{m})$.

$x_{s u}$ : Límite superior para $x$ sobre la esfera $(\mathrm{m})$.

$a$ : Límite superior para $x_{i}$ sobre el plano (m).

0 : Límite inferior para $x_{i}$ sobre el plano (m).

$b$ : Límite superior para $y_{i}$ sobre el plano (m).

0 : Límite inferior para $y_{i}$ sobre el plano (m).

\section{Bibliografía}

Holman, J. P. (1998). Transferencia de calor. España: McGraw-Hill.

Mills, I. (1995). Transferencia de Calor. Colombia: McGraw-Hill.

Halliday, D. (1970). Física, Vol. 2. México: Compañía Editorial Continental.

Lehman, C. H. (1997). Geometría Analítica. México: Limusa.
Carnahan, B. y Luther, B. (1969). Applied Numerical Methods. New York: John Wiley and Sons

Burden, R. (1996). Análisis numérico. México: Grupo Editorial Iberoamericano.

Sobol, I. M. (1983). Método de Montecarlo. Moscú: Mir. 


\section{Apéndice A}

Determinación de los puntos y $\varepsilon\left(x_{\varepsilon}, y_{\varepsilon}, 0\right) \mathbf{y} p^{*}\left(x^{*}, y^{*}, z^{*}\right)$

Mediante el procedimiento dado en seguida se determinaron los puntos

$$
\varepsilon\left(x_{\varepsilon}, y_{\varepsilon}, 0\right) \text { y } p^{*}\left(x^{*}, y^{*}, z^{*}\right)
$$

Determinación del punto $\boldsymbol{\varepsilon}$. La forma simétrica de la ecuación de la recta que pasa por $p$ y $\varepsilon$ es:

$$
\frac{(X-x)}{\left(x-x_{\varepsilon}\right)}=\frac{(Y-y)}{\left(y-y_{\varepsilon}\right)}=\frac{(Z-z)}{(z-0)}
$$

La línea que une $p$ y $\varepsilon$ (figura 3 ) es normal a la esfera y el vector que coincide con esta línea es el vector gradiente calculado en $p$ :

$$
\nabla G=\frac{\partial G}{\partial x} i+\frac{\partial G}{\partial y} j+\frac{\partial G}{\partial z} k
$$

por tanto, los cosenos directores de la recta normal a la esfera son:

$$
\begin{gathered}
\cos \alpha=\frac{1}{|\nabla G|} \frac{\partial G}{\partial x} ; \quad \cos \beta=\frac{1}{|\nabla G|} \frac{\partial G}{\partial y} ; \quad \cos \gamma=\frac{1}{|\nabla G|} \frac{\partial G}{\partial z} \\
\nabla G=2(x-h) i+2(y-k) j+2(z-l) k
\end{gathered}
$$

Conocidos $G$ (ecuación-1) y los puntos por los que pasa la recta $p \varepsilon$, se determinan sus cosenos directores como:

$$
\cos \alpha=\frac{\left(x-x_{\varepsilon}\right)}{p \varepsilon} ; \quad \cos \beta=\frac{\left(y-y_{\varepsilon}\right)}{p \varepsilon} ; \quad \cos \gamma=\frac{(z-0)}{p \varepsilon}
$$

$p \varepsilon:$ distancia entre los dos puntos $p$ y $\varepsilon$ 


$$
p \varepsilon=\sqrt{\left(x-x_{\varepsilon}\right)^{2}+\left(y-y_{\varepsilon}\right)^{2}+z^{2}}
$$

Igualando los cosenos directores entre las ecuaciones (A1) y (A2) se obtienen las coordenadas del punto $\varepsilon$ (figura 3):

$$
x_{\varepsilon}=x-\frac{(x-h) z}{(z-l)} ; \quad y_{\varepsilon}=y-\frac{(y-k) z}{(z-l)} ; \quad z_{\varepsilon}=0
$$

Determinado el punto $\varepsilon$, se calcula la distancia $p \varepsilon$.

Determinación del punto $p^{*}$. Para calcular esta distancia es necesario resolver el siguiente problema geométrico en $\mathbf{R}^{3}$ :

Dados la recta $\mathrm{pp}_{\mathrm{i}}$ (figura 3): $\quad \frac{(X-x)}{\left(x-x_{\varepsilon}\right)}=\frac{(Y-y)}{\left(y-y_{\varepsilon}\right)}=\frac{(Z-z)}{z}$

y el punto exterior a ella $\varepsilon\left(x_{\varepsilon}, y_{\varepsilon}, 0\right)$ se necesita calcular la distancia del punto $\varepsilon$ a la recta $\mathrm{pp}_{\mathrm{i} .}$ El problema se reduce a hallar el punto $p^{*}\left(x^{*}, y^{*}, z^{*}\right)$ (pie de la perpendicular a $\mathrm{pp}_{\mathrm{i}}$ desde $\varepsilon$ figura 3) y después, por la fórmula de distancia euclidiana entre dos puntos, se calcula $d$. Utilizando métodos vectoriales se determinó el siguiente sistema de ecuaciones para hallar $p$ :

$$
\begin{gathered}
z x^{*}-\left(x-x_{i}\right) z^{*}=-z x_{i} \\
z y^{*}-\left(y-y_{i}\right) z^{*}=-z y_{i} \\
z\left(x-x_{i}\right) x^{*}-z\left(y-y_{i}\right) y^{*}-z^{2} z^{*}=z\left(x-x_{i}\right) x_{\varepsilon}-z\left(y-y_{i}\right) y_{\varepsilon}
\end{gathered}
$$

Determinado $p\left(x^{*}, y^{*}, z^{*}\right)$ de la solución del sistema anterior, se calcula $d$

$$
d=\sqrt{\left(x-x_{\varepsilon}\right)^{2}+\left(y-y_{\varepsilon}\right)^{2}+\left(z-z_{\varepsilon}\right)^{2}}
$$

